\title{
Avaliação dos resultados de intervenção após mudanças realizadas nos cuidados peri-operatórios em pacientes submetidos a operações abdominais eletivas
}

\author{
Evaluation of changes made in the peri-operative care in patients submitted to \\ elective abdominal surgery
}

\author{
Mayra da Rosa Martins Walczewski ; Ariane Zanetta Justino ${ }^{2}$; Eduardo André Bracci Walczewski ${ }^{3}$; Tatiane Coan ${ }^{4}$
}

\author{
R E S U M O
}

\begin{abstract}
Objetivo: Avaliar os resultados da introdução de novas medidas visando acelerar a recuperação pós-operatória de pacientes submetidos à cirurgia abdominal eletiva. Métodos: Foram observados e entrevistados 162 pacientes e entrevistados durante dois períodos distintos: o primeiro, entre outubro a dezembro de $2009(n=81)$ formado por pacientes submetidos à condutas convencionais de acompanhamento peri-operatório (período pré-intervenção) e o segundo, entre março a maio de 2010 ( $n=81$ ), formado por um novo grupo de pacientes submetidos, então, ao novo protocolo de condutas de acompanhamento peri-operatório. A coleta de dados nos dois períodos ocorreu sem o conhecimento dos profissionais do serviço. As variáveis observadas foram: indicação de suporte nutricional pré-operatório; tempo de jejum pré e pós-operatório; volume de hidratação; uso de sondas e drenos; tempo de internação e morbidade pós-operatória. Resultados: Na comparação entre os dois períodos, observou-se no período pós-intervenção uma diminuição de 2,5 horas no tempo de jejum pré-operatório ( $p=0,0002)$. Em relação à reintrodução da dieta por via oral houve diferença entre os dois períodos $(p=0,0007)$. Considerando os pacientes que não apresentaram complicações no pósoperatório houve diminuição significativa no tempo de internação ( $p=0,001325$ ). Houve uma redução de aproximadamente $50 \%$ no uso de antibiótico no período pós-intervenção $(p=0,00001)$. Conclusão: A adoção de medidas multidisciplinares peri-operatórias é exequível dentro da nossa realidade e embora não tenha havido alterações com significância estatística no presente estudo, pode melhorar a morbidade e diminuir tempo de internação em cirurgia geral de modo significante.
\end{abstract}

Descritores: Avaliação de resultados (cuidados de saúde). Protocolos. Cuidados pré-operatórios. Cuidados pós-operatórios. Terapia nutricional.

\section{INTRODUÇÃO}

O trato digestório é o responsável pela digestão e absorção dos alimentos. Além dessa importante função dada ao intestino, este funciona também como "um órgão central de estresse" e como uma barreira para evitar que microorganismos e toxinas no lúmen se propaguem para tecidos e órgãos distantes'.

As consequências da desnutrição no pré-operatório foram, pela primeira vez, reconhecidas na década de 1930². Segundo Studley, há uma relação direta no préoperatório entre perda de peso e taxa de mortalidade operatória ${ }^{3}$. Pacientes submetidos à operações gastrintestinais estão em risco de depleção nutricional por inadequada ingestão alimentar, tanto no pré-operatório como no pósoperatório. Por isso, a terapia nutricional vem sendo utilizado há mais de quarenta anos principalmente no cuidado peri-operatório de pacientes cirúrgicos ${ }^{4}$. Múltiplos estudos têm abordado o tema, descrevendo métodos para avaliar o estado nutricional, determinando quais pacientes devem receber apoio nutricional e como os mesmos devem recebê$\mathrm{l}^{5}$. No entanto, alguns aspectos ainda continuam a ser alvo de inúmeras controvérsias que envolvem alguns dos paradigmas existentes em medicina, especialmente em cirurgia, que geram, por sua vez, angústia e medo aos profissionais envolvidos no tratamento de pacientes operados ${ }^{6}$.

A recuperação pós-operatória de pacientes submetidos à operações no aparelho digestivo continua sendo um grande desafio para o cirurgião. Nas operações de maior porte, os índices de complicações mantêm-se alto, variando de 20 a $40 \%^{7}$. No entanto, observa-se que grande parte das rotinas voltadas aos cuidados peri-operatórios em cirurgia abdominal vem se mantendo pouco alteradas ao longo do tempo 6 . Em cirurgia do aparelho digestivo, os principais pontos a serem considerados e analisados dizem

Trabalho realizado no Departamento de Clínica Cirúrgica, Universidade do Sul de Santa Catarina (UNISUL), Brasil.

1. Professora de Gastroenterologia do Curso de Medicina da UNISUL-SC-BR; 2. Médica Clínica Geral da Prefeitura de Palhoça no ESF Cambirela

- Praia de Fora-S-SC-BR; 3. Professor de Cirurgia Geral do Curso de Medicina da UNISUL-SC-BR; 4. Nutricionista do Hospital Nossa Senhora da

Conceição - SC-BR. 
respeito à abordagem nutricional, limitação ao uso de drenos, sonda nasogástrica; menor hidratação venosa perioperatória, e utilização sistemática do preparo pré-operatório do cólon ${ }^{8}$

Atualmente, o grande foco da assistência perioperatória tem sido assegurar adequada ingestão alimentar, assim como, se tem investigado métodos de prestação de apoio nutricional e suas vantagens clínicas em relação à diminuição das alterações metabólicas associadas com o trauma cirúrgico ${ }^{2}$. A resposta orgânica apresenta-se aumentada em pacientes submetidos ao jejum noturno, quando comparada a pacientes que receberam infusão de carboidratos $^{9,10}$. A liberação da dieta no pós-operatório é outro ponto controverso que tem gerado inúmeras discussões. Em geral, os cirurgiões aguardam a resolução do chamado íleo pós-operatório. Essa prática não só pode contribuir com a piora do estado nutricional de pacientes previamente desnutridos, como aumenta o tempo de internação hospitalar ${ }^{11}$. Por outro lado, pacientes submetidos a grandes intervenções e alimentados precocemente apresentam melhor oxigenação da mucosa intestinal ${ }^{12}$, diminuição da resposta orgânica e do número de complicações no pósoperatório ${ }^{13-15}$, assim como, redução do tempo de dismotilidade intestinal ${ }^{16,17}$.

Apesar de importantes avanços nos cuidados cirúrgicos, complicações pós-operatórias são ainda de grande preocupação. Nos últimos anos, tradicionalmente vários aceites de regras e "verdades" em relação a cuidados nutricionais peri-operatórios têm sido contestados. A atual meta tem sido a luta para reduzir as complicações advindas da desnutrição e seus efeitos nocivos em favor do paciente e do sistema de saúde ${ }^{18}$. Reduzir o tempo do jejum pré e pós-operatório está entre as questões que têm atraído mais atenção nos estudos recentes, que nos comprovam a importância do apoio nutricional peri-operatório no que diz respeito à diminuição de morbidade e mortalidade. A diminuição de custos hospitalares pela não necessidade de vários procedimentos também é importante dentro desse contexto.

Com base no exposto, o presente estudo tem como objetivo avaliar os resultados após uma intervenção de novas condutas em cirurgia abdominal, fundamentadas na prática baseada em evidências visando acelerar a recuperação pós-operatória de pacientes submetidos à cirurgias abdominais no Hospital Nossa Senhora da Conceição, visto que, não existe atualmente uma padronização quanto a condutas em cirurgia abdominal.

\section{MÉTODOS}

Foram estudados de modo prospectivo 162 pacientes maiores de 18 anos submetidos à operações abdominais eletivas no Hospital Nossa Senhora da Conceição. O projeto foi submetido e aprovado no Comitê de Ética em Pesquisa da UNISUL sob o registro $n^{\circ}$ 09.395.4.01.III.
Os pacientes foram observados e entrevistados durante dois períodos distintos: o primeiro, entre outubro a dezembro de 2009 ( $n=81$ ) formado por pacientes submetidos a condutas convencionais de acompanhamento perioperatório (período pré-intervenção) e o segundo, entre março a maio de 2010 ( $n=81$ ), formado por um novo grupo de pacientes submetidos, então, ao novo protocolo de condutas de acompanhamento peri-operatório. No intervalo desses dois períodos foi feita uma intervenção onde se realizou um treinamento com exposição de seminários e discussão de artigos sobre o assunto baseado em diretrizes atuais onde são preconizadas novas condutas em cirurgia abdominal. Nesses seminários, foram abordados os seguintes temas: Nutrição peri-operatória; Hidratação venosa peri-operatória; Importância da analgesia e redução de vômitos na redução da resposta metabólica ao trauma; Cuidados com o paciente (informação pré-operatória, drenos, sondas e deambulação ultra-precoce); Evidência contrária ao preparo mecânico do cólon e racionalização do uso de antibióticos em cirurgia. A coleta de dados nos dois períodos ocorreu sem o conhecimento dos profissionais do serviço antes e após o treinamento (cegamento ou mascaramento). Não houve coerção ao uso do novo protocolo. Os cirurgiões tiveram livre arbítrio para optarem pelos cuidados peri-operatórios em seus pacientes.

A tabela 1 mostra o conjunto de medidas estabelecidas segundo o treinamento realizado durante a intervenção e as condutas convencionais que vinham sendo aplicadas antes da implantação da mesma. As operações foram divididas conforme o potencial de complicações em porte I (operações envolvendo parede abdominal e laparotomias sem abertura de alças e/ou manipulação de vias biliares) e porte II (operações envolvendo laparotomias com abertura de alças e/ou manipulação de vias biliares).

As variáveis coletadas em relação aos períodos pré e pós-intervenção foram: indicação e realização de suporte nutricional pré-operatório; tempo de jejum pré e pós-operatório; volume de hidratação venosa no pós-operatório; dia de re-alimentação por via oral ou enteral no pós-operatório; uso de dreno cavitário (drenagem aberta ou fechada) e sonda nasogástrica no pós-operatório; tempo de internação pós-operatório; uso de antibiótico. Visando avaliar o impacto de tais medidas na morbidade cirúrgica dos pacientes analisou-se nos dois grupos a morbidade (com ênfase na infecção do sitio cirúrgico) e o tempo de internação pós-operatório. As variáveis foram analisadas em relação ao total de operações realizadas $(n=162)$, e ao total de cirurgias porte $\|(n=51)$.

Para análise de dados foi utilizado o pacote estatístico SPSS 12. As variáveis contínuas foram descritas por medidas de tendência central e dispersão e testadas através do teste t-Student ou Kruskal-Wallis, quando apropriado. As variáveis qualitativas foram testadas através do teste qui-quadrado ou exato de Fisher, quando apropriado. A força de associação entre os desfechos foi realizada atra- 
Tabela 1 - Condutas em cirurgia abdominal aplicadas na enfermaria de cirurgia geral do HNSC antes e depois da Intervenção, preconizadas pelo Projeto ACERTO modificadas.

\section{Condutas convencionais de acompanhamento peri- operatório}

- Jejum pré-operatório mínimo de 8h (desde a noite anterior ao ato operatório).

- Liberação da dieta pós-operatória após eliminação de flatos ou evacuação (saída de "íleo").

- Hidratação venosa no pós-operatório no volume de $40 \mathrm{ml} /$ kg.

- Preparo mecânico sistemático do cólon para operações.

- colorretais com manitol ou fosfo-soda, por lavagens retais seriadas.

- Uso de drenos, sondas e antibióticos conforme preferência do cirurgião.

- Mobilização pós-operatória precoce.

\section{Novo protocolo de condutas de acompanhamento peri-operatório.}

- Não permitir um jejum prolongado no pré-operatório. Indicar uso de dieta líquida enriquecida com carboidrato até na véspera da operação, podendo a ingesta acontecer ate 2 horas antes da operação. Exceção: obeso mórbido, refluxo gastresofágico importante e síndrome de estenose pilórica.

- Em cirurgias da via biliar, herniorrafias e afins, dieta oral liquida oferecida no mesmo dia da operação (6-12 horas após).

- Em operações com anastomose digestiva re-introdução de dieta no $1^{\circ} \mathrm{PO}$ (dieta liquida) ou no mesmo dia da operação.

- Em cirurgias com anastomose esofágica, dieta no $1^{\circ} \mathrm{PO}$ pela jejunostomia ou sonda naso-entérica

- Hidratação endovenosa não deve ser prescrita em herniorrafias no PO imediato. Hidratação endovenosa deve ser retirada com 12 horas após colecistectomias salvo exceções.

- Salvo exceções, nas demais, reposição volêmica ate o $1^{\circ} \mathrm{PO}$ no máximo $30 \mathrm{ml} / \mathrm{Kg} /$ dia.

- Não realizar o preparo de cólon de rotina para cirurgias colorretais no pré-operatório.

- Não usar drenos e sondas de rotina. Uso racional e padronizado de antibióticos*.

- Informar ao paciente antes da operação detalhes do procedimento a ser realizado, encorajando-o a deambular e realimentar precocemente no $\mathrm{PO}$.

* Conforme protocolo preconizado por Ferraz e Ferraz ${ }^{19}$.

vés do risco relativo com respectivo intervalo de confiança de $95 \%(p<0,05)$.

\section{RESULTADOS}

Ao longo do tempo de observação, foram incluídos no nosso estudo 162 pacientes submetidos à laparotomia com intervenção sobre órgãos do aparelho digestivo ou operações de parede abdominal conforme pode ser observado na tabela 2 (111 operações porte I e 51 operações porte II), tendo havido dois óbitos no pósoperatório (índice de mortalidade global de 1,2\%), um em cada período de estudo $(p=1,0)$. Igualmente em cada período foram operados 81 pacientes.

\section{tória}

Terapia de suporte nutricional pré-opera-

Vinte pacientes apresentaram percentual de perda de peso de $10 \%$ e foram considerados em risco nutricional, não houve diferença entre os dois grupos. Ob- servou-se também que não houve diferença significativa quanto aos pacientes que receberam suporte nutricional entre os 2 períodos (7,4\% vs 9,9\%; $p=0,57)$ (Tabela 3).

\section{Jejum e hidratação peri-operatórios}

Constatou-se que os pacientes ficavam, em média, 14 horas em jejum e muito mais, portanto, do que as preconizadas oito horas de jejum pré-operatório antes da intervenção. Na comparação entre os dois períodos, observou-se no período pós-intervenção uma diminuição de 2,5 horas no tempo de jejum pré-operatório (14 [5-28] horas vs. 10 [5-24] horas; $p=0,0002$ ). Considerando apenas operações porte II, houve queda de 1,5 horas no tempo de jejum pré-operatório (12 [5-24] horas vs 13 [5-17] horas; $p=0,24$ ) no período pós-intervenção em relação ao período pré-intervenção. Em relação à reintrodução da dieta por via oral houve diferença entre os dois períodos (1 [1-2] dias vs. 1 [1-2] dias; $p=0.0007$ ), diferente do volume de fluidos intravenosos infundidos por paciente que não houve alteração estatística (4 [1-23] litros vs 4 [1-28] litros; $p=0,66)$. Esses dados podem observados na tabela 4 . 
Tabela 2 - Operações realizadas segundo os períodos estudados.

\begin{tabular}{lcc}
\hline Operações & Pré-Intervenção & Pós-intervenção \\
\hline Duodenopancreatectomia & 0 & 3 \\
Gastrectomia subtotal & 1 & 2 \\
Exploração das vias biliares & 0 & 6 \\
Laparotomia exploradora & 12 & 11 \\
Cirurgia de obesidade mórbida & 1 & 0 \\
Operações com anastomose instestinal* & 17 & 8 \\
Herniorrafias & 16 & 23 \\
Colecistectomia & 34 & 28 \\
Total & $\mathbf{8 1}$ & $\mathbf{8 1}$
\end{tabular}

*Colectomia, Amputação Abdomino-perineal do reto, Abaixamento colorretal, Fechamento de Colostomia e Enteroanastomose.

\section{Drenos e sondas}

Em relação ao uso de drenos cavitários não foram observadas diferenças estatísticas entre os pacientes operados nos dois períodos ( $42 \%$ vs $32,1 \% ; p=0,19)$, assim também como o uso de sonda nasogástrica $(6,2 \%$ vs $7,4 \% ; p=0,75)$.

\section{Tempo de internação pós-operatória}

Não houve diferença estatística nos períodos observados (3 [1-13] dias vs $2[1-11]$ dias; $p=0,09$ ). Considerando os pacientes que não apresentaram complicações no pós-operatório houve diminuição significativa no tempo de internação (3 [1-13] dias vs 2 [1-10]; $p=0,001325)$.

\section{Uso de antibiótico}

Houve uma redução de aproximadamente $50 \%$ no uso de antibiótico no período pós-intervenção ( $R R=0,50$; IC 95\%: 69,1\% vs 34,6\%); $p=0,00001$ ).
Morbidade pós-operatória

Em relação aos percentuais de infecção do sítio cirúrgico $(1,2 \%$ vs $2,5 \%$; $p=0,56)$ e de complicações pósoperatórias $(3,7 \%$ vs $4,9 \% ; p=0,70)$ não houve diferença estatística em ambos os períodos observados. Da mesma forma ocorreu considerando apenas operações porte II em relação à infecção do sítio cirúrgico $(3,6 \%$ vs $8,3 \% ; p=0,44)$ e complicações pós-operatórias (7,1\% vs 16,7\%; $p=0,26)$. Dentre as complicações pós-operatórias houve seis casos de deiscências anastomóticas (fístulas pós-operatórias), sendo dois na primeira fase e quatro na segunda fase, considerando apenas operações porte $\|(p=0,26)$.

\section{Preparo mecânico do cólon}

Foram realizadas 17 operações colorretais no período pré-intervenção e oito no segundo período do estudo. O preparo do cólon continuou sendo realizado no período pós-intervenção.

Tabela 3 - Caracterização clínica e epidemiológica dos grupos de pacientes estudados nos períodos Pré e Pós-Intervenção $(\mathrm{N}=162)$.

\begin{tabular}{|c|c|c|c|c|c|}
\hline \multirow[b]{2}{*}{ Sexo } & \multicolumn{2}{|c|}{ Período Pré-Intervenção $(n=81)$} & \multicolumn{2}{|c|}{ Período Pós-Intervenção $(n=81)$} & \\
\hline & $\begin{array}{l}\text { Masculino } \\
39(44,4 \%)\end{array}$ & $\begin{array}{c}\text { Feminino } \\
45(55,6 \%)\end{array}$ & $\begin{array}{l}\text { Masculino } \\
42(51,9 \%)\end{array}$ & $\begin{array}{c}\text { Feminino } \\
39(48,1 \%)\end{array}$ & \\
\hline Perda Ponderal > 10\% & \multicolumn{2}{|c|}{$52,67( \pm 17,66)$} & \multicolumn{2}{|c|}{$50,98( \pm 17,24)$} & $p=0,53$ \\
\hline nos últimos 6 meses & \multicolumn{2}{|c|}{$12(14,8 \%)$} & \multicolumn{2}{|c|}{$8(9,9 \%)$} & $p=0,34$ \\
\hline IMC & $\begin{array}{r}43(53 \\
29(35,8 \\
6(7,4 \%) \\
1(1,2 \%) \\
2(2,5 \%)\end{array}$ & $\begin{array}{l}\text { Vormal } \\
\text { brepeso } \\
\text { lade grau I } \\
\text { ade grau ÏI } \\
\text { ade grau III }\end{array}$ & $\begin{array}{r}63(7) \\
17(21 \\
1(1,2 \%)\end{array}$ & $\begin{array}{l}\text { lormal } \\
\text { repeso } \\
\text { ade grau I }\end{array}$ & $p=0,009$ \\
\hline Solução Glicosada Pré-operatóri & \multicolumn{2}{|c|}{$38(46,9 \%)$} & \multicolumn{2}{|c|}{$31(38,3 \%)$} & $p=0,26$ \\
\hline Suporte Nutricional & & & \multicolumn{2}{|c|}{$8(9,9 \%)$} & $p=0,57$ \\
\hline NPT & \multicolumn{2}{|c|}{$5(6,2 \%)$} & \multicolumn{2}{|c|}{$2(2,5 \%)$} & $p=0,24$ \\
\hline Porte das operações & $\begin{array}{c}\text { Porte I } \\
54(66,7 \%)\end{array}$ & $\begin{array}{c}\text { Porte II } \\
27(33,3 \%)\end{array}$ & $\begin{array}{c}\text { Porte I } \\
57(70,4 \%)\end{array}$ & $\begin{array}{c}\text { Porte II } \\
24(29,6 \%)\end{array}$ & $p=0,61$ \\
\hline
\end{tabular}


Tabela 4 - Abordagem nutricional peri-operatória, hidratação endovenosa pós-operatória, uso de drenos e sonda nasogástrica, uso de antibiótico nos períodos anterior e posterior à intervenção.

\begin{tabular}{|c|c|c|c|c|}
\hline & Operações & Pré-Intervenção & Pós-Intervenção & $p$ \\
\hline \multirow[t]{2}{*}{ Tempo de jejum pré-operatório (horas) } & Porte I e II & $14[5-28]$ & $10[5-24]$ & 0,0002 \\
\hline & Porte II & $12[5-24]$ & $13[5-17]$ & 0,24 \\
\hline \multirow[t]{2}{*}{ Dia de pós-operatório de re-introdução da dieta } & Porte I e II & $1 \quad[1-2]$ & $1 \quad[1-2]$ & 0,0007 \\
\hline & Porte ॥ & $1 \quad[1-2]$ & {$[1-2]$} & 0,0016 \\
\hline \multirow{2}{*}{$\begin{array}{l}\text { Volume de hidratação endovenosa no } \\
\text { pós-operatório (litros) }\end{array}$} & Porte I e II & $4[1-23]$ & $4 \quad[1-28]$ & 0,66 \\
\hline & Porte II & $8[1-23]$ & $9,5[4-28]$ & 0,10 \\
\hline \multirow[t]{2}{*}{ Uso de drenos cavitários } & Porte I e II & $34 \quad(42 \%)$ & $(32,1)$ & 0,19 \\
\hline & Porte II & $(64,3 \%)$ & $(83,3 \%)$ & 0,12 \\
\hline \multirow[t]{2}{*}{ Uso de sonda nasogástrica } & Porte I e I| & $(6,2 \%)$ & $(7,4 \%)$ & 0,75 \\
\hline & Porte ॥ & $(10,7 \%)$ & $(25,0 \%)$ & 0,16 \\
\hline Uso de antibiótico & Porte I e II & $56 \quad(69,1 \%)$ & $(34,6 \%)$ & 0,00001 \\
\hline
\end{tabular}

\section{DISCUSSÃO}

A aplicação de programas padronizados objetivando a otimização da recuperação pós-operatória tem apresentado bons resultados ${ }^{6}$. Estudos clínicos consistentes têm feito com que velhos paradigmas sejam questionados e substituídos por práticas mais modernas, respaldadas em evidência 20 .

A nutrição tanto no pré como no pós-operatório tem papel relevante, diminuindo a resposta orgânica ao estresse, interferindo de maneira significativa na evolução dos pacientes ${ }^{6}$. Neste trabalho pôde-se observar que, com os cuidados implantados, foi possível a redução do tempo de jejum peri-operatório, com uma significância estatística maior em relação ao período de jejum pós-operatório. Conjuntamente, houve também uma discreta diminuição da infusão endovenosa de fluidos, sem que isso alterasse negativamente os resultados cirúrgicos. Entretanto, os resultados mostraram que os pacientes ainda ficaram mais de dez horas em jejum aguardando uma operação. Isso mostra que houve melhora, porém, pelo que se preconiza hoje na literatura, este tempo ainda está prolongado.

Com relação à morbidade global e infecção do sítio cirúrgico observou-se uma pequena elevação no percentual estatístico observado, tanto na casuística global quanto nas operações de maior porte. Embora nesse estudo não tenha sido verificada redução da morbimortalidade nos pacientes operados, em comparação a outros trabaIhos pôde-se observar uma menor taxa de complicações pós-operatórias e redução no tempo de internação hospitalar $^{8}$. Assim, a adoção de um programa multidisciplinar com medidas cientificamente embasadas visando melhorar a recuperação pós-operatória relacionou-se como uma redução do tempo de internação pós-operatória.

Ferraz et a/ ${ }^{19}$ preconizam o uso racional e padronizado de antibióticos em cirurgia. Houve uma importante redução estatística em relação ao emprego de antibióticos na segunda fase do estudo. Deve ser considerada, tam- bém, nos resultados encontrados a importância de outros pontos contemplados nesse projeto, tais como controle efetivo da dor e vômitos pós-operatórios, informação mais detalhada do procedimento ao paciente diminuindo sua ansiedade, mobilização ultraprecoce e a interação multidisciplinar. Isso aliado à redução de fluidos e tempo de internação representa diminuição de gastos hospitalares.

É conhecida a importância do suporte nutricional pré-operatório para pacientes desnutridos, porém, mesmo com normas que preconizam o suporte nutricional pré-operatório nesses doentes, ele não era empregado na maioria dos $\operatorname{casos}^{6}$. Pode-se observar que antes da intervenção cirúrgica nem todos os pacientes desnutridos receberam suporte nutricional, o inverso ocorreu na segunda fase do estudo, onde todos receberam suporte nutricional.

O jejum pré-operatório prolongado, habitualmente entre seis e oito horas, é prática aceita desde a introdução da anestesia, em 1840. Isso ocorria devido à garantia do esvaziamento gástrico, evitando assim a broncoaspiração no momento da indução anestésica. Contudo, esta conduta tem sido questionada, pois parece não haver evidência de que uma diminuição do período de jejum para líquido em comparação ao regime convencional determine risco de aumento de aspiração pulmonar ou de morbidade relacionada com este evento ${ }^{21}$. Soma-se a este argumento constatações de que o jejum pré-operatório, além de bastante desconfortável e desnecessário, pode ser prejudicial ao potencializar ou perpetuar a resposta orgânica ao trauma ${ }^{22}$. Atualmente as sociedades de anestesia recomendam regras mais liberais em relação ao jejum, permitindo o uso de líquidos claros até duas horas antes da operação. Estudos mais recentes indicam que o uso de uma solução de líquido enriquecida com carboidrato determinaria maior satisfação, menor irritabilidade, menor número de vômitos, aumento do $\mathrm{pH}$ gástrico e, especialmente, uma menor resposta orgânica ao estresse cirúrgico ${ }^{23}$. Embora o tempo de jejum diminuísse neste estudo não se utilizou 
bebidas com $\mathrm{CHO}$ e nem redução importante do tempo de jejum.

A liberação da dieta e o tipo de alimentos permitidos (líquidos claros progredindo até alimentos sólidos) após a resolução do íleo não têm sido apoiados pela luz da Medicina Baseada em Evidências. O jejum pós-operatório faz parte dos cuidados de rotina prescritos até que haja eliminação de flatos ou de fezes. Isso, em geral, ocorre em torno do terceiro ou quarto dia pós-operatório. Logo, o tempo de internação hospitalar é maior, pois se aguarda que o doente se alimente e tolere a dieta para ter alta ${ }^{6}$. O impacto da dieta precoce na recuperação pós-operatória e todos esses paradigmas têm sido desafiados e questionados. Alguns estudos têm mostrado que, após a realização de operações colorretais, é possível alimentar os doentes precocemente e dar alta no $2^{\circ}$ e $3^{\circ}$ dias pós-operatórios ${ }^{24}$. Essa prática diminui o desconforto dos doentes, o tempo de internação e, consequentemente, os custos hospitalares ${ }^{6}$. No nosso estudo, o retorno da dieta deu-se com sucesso no primeiro dia de PO na segunda fase do estudo. Essa medida além de não ter sido prejudicial, juntamente com outras adotadas, foi responsável em uma casuística global por melhores resultados no período pós-intervenção.

Em relação à hidratação venosa em pacientes cirúrgicos submetidos à operações eletivas, as evidências apontam que o balanço positivo de água e sódio prejudica o retorno das funções gastrintestinais no pós-operatório, assim como, afetam o organismo como um todo, determinando aumento do período de íleo, da morbidade e mortalidade ${ }^{25}$. Este estudo corrobora na idéia de efetiva redução da carga hídrica administrada no pós-operatório de cirurgias abdominais eletivas. Neste estudo, observouse uma discreta diminuição da quantidade de fluidos administrados de forma parenteral. Entretanto, isso foi notável nas operações de menor porte. Ressalta-se além dos pontos já levantados, a importância da relação desta questão com custos, com a mobilização precoce do paciente (sem cateteres de reposição hídrica o paciente sente-se em melhores condições para se movimentar livremente) e com o estímulo (sede) ao retorno à alimentação por via oral ${ }^{8}$.

Observamos uma discreta alteração em relação à frequência de uso de sonda nasogástrica e drenos cavitários. Acreditamos que drenos na cavidade abdominal representam um enorme impedimento para o paciente movimentar-se e ter alta mais precoce. Algumas metanálises têm demonstrado que o uso de drenos em operações envolvendo anastomoses colônicas não reduz a incidência de complicações nem a gravidade de deiscências de anastomoses ${ }^{26}$. O uso de sondas nasogástricas como rotina também não tem suporte na evidência e acredita-se que podem até mesmo prejudicar o paciente no pós-operatório $^{8}$. Seu uso tem sido desestimulado em muitos serviços de cirurgia ${ }^{27}$. Analisando nossos resultados, o uso de sonda nasogástrica foi da ordem de 7,4\%, relativamente baixo, embora tenha sido utilizada com maior frequência no período pós-intervenção. O mesmo não pode ser dito em relação ao uso de drenos cavitários, utilizado em 34\% das operações da primeira fase do estudo vs $26 \%$ na segunda fase. Notou-se, portanto, uma redução na sua utilização. Acreditamos que a maior utilização de drenos deve-se ao pensamento dos cirurgiões de utilizá-los na tentativa de diagnosticar precocemente complicações, sobretudo as anastomóticas, ou diminuir as suas repercussões.

Atualmente, vem crescendo o número de estudos que envolvem mudanças nas rotinas peri-operatórias de pacientes cirúrgicos. No presente estudo, essas mudanças foram conduzidas por práticas baseada em evidências que vêm sendo introduzidas também por outros serviços de cirurgia geral, que, assim como nós, acreditam que fará em breve parte do dia a dia das enfermarias de cirurgia de instituições do nosso país.

Em conclusão, a adoção de medidas multidisciplinares peri-operatórias é exequível dentro da nossa realidade e, embora não tenha havido alterações com significância estatística no presente estudo, pode melhorar a morbidade e diminuir tempo de internação em cirurgia geral de modo significante.

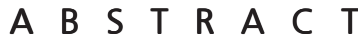

Objective: To evaluate the results of the introduction of new measures to accelerate the postoperative recovery of patients undergoing elective abdominal surgery. Methods: We observed 162 patients and interviewed them on two distinct periods: the first between October to December 2009 ( $n=81)$ comprised patients who underwent conventional perioperative monitoring (preintervention) and the second between March and May $2010(n=81)$, formed by a new group of patients, submitted to the new protocol of perioperative monitoring. Data collection in the two periods occurred without the knowledge of the professionals in the service. The variables were: indication for preoperative nutritional support, duration of fasting, post-operative volume of hydration, use of catheters and drains, length of stay and postoperative morbidity. Results: when comparing the two periods we observed a decrease of 2.5 hours in the time of preoperative fasting $(p=0.0002)$ in the post-intervention group. As for the reintroduction of oral diet, there was no difference between the two periods $(p=0.0007)$. When considering the patients without postoperative complications, there was a significantly decreased length of stay $(p=0.001325)$. There was a reduction of approximately 50\% in antibiotic use in the post-intervention group $(p=0.00001)$. Conclusion: The adoption of multidisciplinary perioperative measures is feasible within our reality, and although there was no statistically significant changes in the present study, it may improve morbidity and reduce length of stay in general surgery.

Key words: Outcome assessment (health care). Protocols. Preoperative care. Postoperative care. Nutrition therapy. 


\section{REFERENCIAS}

1. IMeN - Instituto de Metabolismo e Nutrição. Dilemas - nutrição parenteral x nutrição enteral. Acessado em agosto de 2009. Disponível em: http://www.nutricaoclinica.com.br/2005080227/ terapia-nutricional-parenteral/dilemas-nutricao-parenteral-xnutricao-enteral

2. Ward N. Nutrition support to patients undergoing gastrointestinal surgery. Nutr J. 2003;2:18.

3. Studley HO. Percentage of weight loss. A basic indicator of surgical risk in patients with chronic peptic ulcer. JAMA. 1936;106(6):45860.

4. Jiang $\mathrm{XH}$, Li N, Li JS. Intestinal permeability in patients after surgical trauma and effect of enteral nutrition versus parenteral nutrition. World J Gastroenterol. 2003:9(8):1878-80.

5. Salvino RM, Dechicco RS, Seidner DL. Perioperative nutrition support: who and how. Cleve Clin J Med. 2004;71(4):345-51.

6. Correia MITD, Silva RG. Paradigmas e evidências da nutrição perioperatória. Rev Col Bras Cir. 2005;32(6):342-7.

7. Lång $\mathrm{M}$, Niskanen $\mathrm{M}$, Miettinen $\mathrm{P}$, Alhava $\mathrm{E}$, Takala J. Outcome and resource utilization in gastroenterological surgery. Br J Surg. 2001:88(7):1006-14

8. Aguilar-Nascimento JE, Bicudo-Salomão A, Caporossi C, Silva RM, Cardoso EA, Santos TP. Acerto pós-operatório: avaliação dos resultados da implantação de um protocolo multidisciplinar de cuidados peri-operatórios em cirurgia geral. Rev Col Bras Cir. 2006;33(3):181-8.

9. Ljungqvist $O$, Thorell A, Gutniak M, Häggmark T, Efendic S. Glucose infusion instead of preoperative fasting reduces postoperative insulin resistance. J Am Coll Surg. 1994;178(4):329-36.

10. Nygren J, Soop M, Thorell A, Efendic S, Nair KS, Ljungqvist $O$ Preoperative oral carbohydrate administration reduces postoperative insulin resistance. Clin Nutr. 1998;17(2):65-71.

11. DiFronzo LA, Yamin N, Patel K, O'Connell TX. Benefits of early feeding and early hospital discharge in elderly patients undergoing open colon resection. J Am Coll Surg. 2003;197(5):747-52.

12. Braga M, Gianotti L, Gentilini O, Liotta S, Di Carlo V. Feeding the gut early after digestive surgery: results of a nine-year experience. Clin Nutr. 2002;21(1):59-65.

13. Enzi G, Casadei A, Sergi G, Chiarelli A, Zurko F, Mazzoleni F. Metabolic and hormonal effects of early nutritional supplementation after surgery in burn patients. Crit Care Med. 1990;18(7):719-21.

14. Kudsk KA. Gut mucosal nutritional support-enteral nutrition as primary therapy after multiple system trauma. Gut. 1994;35(1 Suppl):S52-4.

15. Moore FA, Feliciano DV, Andrassy RJ, McArdle AH, Booth FV, Morgenstein-Wagner TB, et al. Early enteral feeding, compared with parenteral, reduces postoperative septic complications. The results of a meta-analysis. Ann Surg. 1992;216(2):172-83.

16. Di Fronzo LA, Cymerman J, O'Connell TX. Factors affecting early postoperative feeding following elective open colon resection. Arch Surg. 1999;134(9):941-5; discussion 945-6.
17. Waldhausen JH, Shaffrey ME, Skenderis BS 2nd, Jones RS, Schirmer BD. Gastrointestinal myoelectric and clinical patterns of recovery after laparotomy. Ann Surg. 1990;211(6):777-84; discussion 785

18. Akbarshahi $H$, Andersson $B$, Nordén $M$, Andersson R. Perioperative nutrition in elective gastrointestinal surgery-potential for Improvement ?. Dig Surg. 2008;25(3):165-74.

19. Ferraz EM, Ferraz AAB. Antibioticoprofilaxia. In: Ferraz EM, editor. Infecção em cirurgia. Rio de Janeiro: Medsi; 1997. p.345-52.

20. Nygren J, Hausel J, Kehlet H, Revhaug A, Lassen K, Dejong C, et al. A comparison in five European Centers of case mix, clinical management and outcomes following either conventional or fasttrack perioperative care in colorectal surgery. Clin Nutr. 2005;24(3):455-61.

21. Pearse $R$, Rajakulendran $Y$. Pre-operative fasting and administration of regular medications in adult patients presenting for elective surgery. Has the new evidence changed practice ?. Eur J Anaesthesiol. 1999;16(8):565-8.

22. Ljungqvist $\mathrm{O}, \mathrm{S}$ A reide $\mathrm{E}$. Preoperative fasting. $\mathrm{Br} \mathrm{J}$ Surg 2003;90(4):400-6.

23. Nygren J, Thorell A, Ljungqvist O. Preoperative oral carbohydrate nutrition: an update. Curr Opin Clin Nutr Metab Care. 2001:4(4):255-9.

24. Basse L, Thorb/EI JE, LÆEss| K, Kehket H. Colonic surgery with accelerated rehabilitation or conventional care. Dis Colon Rectum. 2004;47(3):271-7; discussion 277-8. Erratum in: Dis Colon Rectum. 2004;47(6):951. Dis Colon Rectum. 2005;48(8):1673.

25. Macafee DA, Alisson SP, Lobo DN. Some interactions between gastrointestinal function and fluid and electrolyte homeostasis Curr Opin Clin Nutr Metab Care. 2005;8(2):197-203.

26. Fearon KC, Ljungqvist O, Von Meyenfeldt M, Revhaug A, Dejong $\mathrm{CH}$, Lassen $\mathrm{K}$, et al. Enhanced recovery after surgery: a consensus review of clinical care for patients undergoing colonic resection. Clin Nutr. 2005:24(3):466-77.

27. Cheatham ML, Chapman WC, Key SP, Sawyers JL. A meta-analysis of selective versus routine nasogastric decompression after elective laparotomy. Ann Surg. 1995;221(5):469-76; discussion 476-8.

Recebido em 08/06/2011

Aceito para publicação em 18/08/2011

Conflito de interesse: nenhum

Fonte de financiamento: nenhum

\section{Como citar este artigo:}

Walczewski MRM, Justino AZ, Walczewski EAB, Coan T. Avaliação dos resultados de intervenção após mudanças realizadas nos cuidados peri-operatórios em pacientes submetidos à cirurgias abdominais eletivas. Rev Col Bras Cir. [periódico na Internet] 2012; 39(2). Disponível em URL: http://www.scielo.br/rcbc

\section{Endereço para correspondência:}

Mayra Martins Walczewski

E-mail: mayrarmartins@hotmail.com 